\title{
The structure of the Akn 120 emitting region: The line shapes and long-term $\mathrm{H}_{\beta}$ line profile variation
}

\author{
L. Č. Popović, N. Stanić, A. Kubičela, and E. Bon \\ Astronomical Observatory, Volgina 7, Belgrade, Yugoslavia
}

Received 9 October 2000 / Accepted 6 December 2000

\begin{abstract}
To investigate the structure of the Akn 120 emitting region we have analysed the spectral line profiles of $\mathrm{H}_{\alpha}, \mathrm{H}_{\beta}, \mathrm{Ly}_{\alpha}$, C III[1909], C IV[1549] and Mg II[2798] lines using a Gaussian-decomposition approach. The decomposed shape of the Akn 120 lines indicates three separated broad line-emitting regions, in two of them, the systematic motions of emitting gas are present. It seems that one of the two regions has a high random velocity of emitters $\left(\approx 2500 \mathrm{~km} \mathrm{~s}^{-1}\right)$ and approaching line-of-sight velocities, and the other has a smaller random velocity of emitters $\left(\approx 1000 \mathrm{~km} \mathrm{~s}^{-1}\right)$ and receding line-of-sight velocities. Also, we discuss the long-term $\mathrm{H}_{\beta}$ line shape variation in the spectra of Akn 120. The $\mathrm{H}_{\beta}$ line (from 96 spectra) was fitted with three broad and one narrow Gaussians through the entire period considered (1977-1990). The central broad as well as blue component of $\mathrm{H}_{\beta}$ and shelf (Fe II template) cause the $\mathrm{H}_{\beta}$ line shape variation. Besides the Gaussian decomposition of the shapes of the three hydrogen lines we propose here a disk model with two more regions located symmetrically to the disk plane. Using the fitting method with the disk model we obtained the parameters of the disk: $i=10^{\circ}$; $R_{\mathrm{inn}} \approx 120-200 R_{\mathrm{g}}, R_{\text {out }} \approx 1000 R_{\mathrm{g}}$.
\end{abstract}

Key words. galaxies: individual (Arakelian 120) - galaxies: Seyfert - line: profiles - accretion, accretion disks

\section{Introduction}

The spectral line investigation of Active Galactic Nuclei (AGNs) helps us to understand physical properties and kinematics of emitting gas. The AGN radiation comes from a compact very luminous center of a galaxy, and in their spectra narrow and broad emission lines are observed. These lines come from a very extensive emitting line region, where plasma has very different parameters; the electron density can range from $10^{2} \mathrm{~cm}^{-3}$ to $10^{11} \mathrm{~cm}^{-3}$ and electron temperatures from several hundreds to tens of thousands Kelvin (see e.g. Osterbrock 1990). It is widely accepted that the "central engine" consists of a massive black hole fueled by an accretion disk. Also, observations in a wide band of wavelengths (X, UV, optical) indicate that an accretion disk should be present as a central part of the majority of AGNs.

Emission line profiles of Active Galactic Nuclei usually show variabilities. Investigations of these variations could provide information about the structure, size and kinematics of the emitting gas in the central part of these objects (see e.g. Foltz et al. 1983; Peterson et al. 1985, 1989, 1998; Peterson \& Gaskell 1991; Peterson 1993; Winge et al.

Send offprint requests to: L. Č. Popović, e-mail: lpopovic@aob.bg.ac.yu
1996; Wandel et al. 1999, etc.). According to the standard model, the broad emission lines observed in spectra of AGNs originate in the high-velocity gas of the surrounding Broad Line Region (BLR) ionized by the UV and optical radiation of the compact central source. Fluctuation in the ionizing continuum cause the variations in emission line fluxes. Also, the structure of the BLR may be investigated through the analysis of the broad emission line profiles.

One of the Sy1 galaxies which has a large-amplitude variability in continuum and in spectral line fluxes and shapes is Akn120 (Peterson et al. 1985; Peterson \& Cota 1987; Peterson et al. 1985, 1989, 1998; Korista 1992; Winge et al. 1996; Popović et al. 1998a; Doroshenko et al. 1999; Wandel et al. 1999). This galaxy has been monitored several times (see e.g. Peterson et al. 1998) in order to investigate complex variations in continuum flux and broad line shape. These investigations show that the integrated flux in the broad emission lines varies very much (Peterson et al. 1989; Winge et al. 1996) and that the BLR is very small (Peterson et al. 1998; Wandel et al. 1999; Doroshenko et al. 1999). The analyses of emission line shapes of Akn 120 have shown that Balmer lines are complex in structure and variable (Korista 1992). The decomposition of $\mathrm{H}_{\beta}$ and other broad lines indicates the 
stratification in the BLR (Korista 1992). The complex and strong profile variations of broad lines of Akn 120 indicate very dynamic processes in the occuring the emitting gas.

In order to investigate the structure of the Akn 120 emitting region, we have analysed the shapes of $\mathrm{H}_{\alpha}$, $\mathrm{H}_{\beta}, \mathrm{Ly}_{\alpha}, \mathrm{CIII}[1909], \mathrm{CIV}[1549]$ and $\mathrm{MgII}[2789]$ lines. Moreover, $\mathrm{H}_{\beta}$ line profile variation of Akn 120 is presented from a set of 96 spectra observed at Crimean Astrophysical Observatory (CrAO) in the period from 1971 till 1990. Also, we have applied a disk model to modeling the complex hydrogen line shapes.

\section{The observations and reduction procedure}

We have analyzed the spectra taken from two sources: 1) The 96 spectra of the $\mathrm{H}_{\beta}$ line in the wavelength interval 4500-5300 $\AA$ that were observed at the Crimean Astrophysical Observatory (CrAO) by K. K. Chuvaev on the $2.6 \mathrm{~m}$ Shain telescope during the period 19711990 (HJD 2443435 till 2447944) and 2) Ly ${ }_{\alpha}$, CIII[1909], CIV[1549] and Mg II[2798] observed by the Hubble Space Telescope in 1994.

The spectra of $\mathrm{Ly}_{\alpha}, \mathrm{CIII}[1909], \quad \mathrm{CIV}[1549]$ and Mg II[2798] lines have been elaborated by HST staff, while all spectra of $\mathrm{H}_{\beta}$ were scanned on a two-coordinate $\mathrm{CrAO}$ microphotometer (Popović et al. 1998a; Doroshenko et al. 1999). Further treatment, including the correction for the film and sky background and instrumental spectral sensitivity was performed. The wavelength and flux calibration were made using the SPE data reduction package, developed by S. G. Sergeev. Wavelength calibration was based on the night sky lines and narrow emission lines of the galaxy. The redshift of Akn120 was taken to be $z=0.03248$ (Foltz et al. 1983).

In order to investigate the long-term variability of the $\mathrm{H}_{\beta}$ line shape and to take into consideration the gaps in observations, spectra were divided into 10 groups (see Table 1).

The spectra within the groups were averaged. In the mean spectra, noises and some short-term variability are to some extent suppressed and possible long-lasting features better expressed. The local continuum in four narrow zones around 4580, 4900, 5270 and $5630 \AA$ was interpolated with a second-degree polynomial. The mean spectra were normalized to the OIII [5007] $\AA$ emission line flux. Notice that the narrow OIII lines (especially OIII 4959) are unusually weak in Akn 120, hence flux calibration based on the OIII line is less reliable than it normally would be.

The broad $\mathrm{H}_{\beta}$ line has two peaks shifted blueward and redward from the line center defined by the narrow emission lines. The peaks are more or less clearly resolved. The $\mathrm{H}_{\beta}$ is blended with $\mathrm{OIII}[4959,5007]$ and some other emission lines (Jackson \& Browne 1989; Meyers \& Peterson 1985; Korista 1992).

\section{The method of analysis}

In order to decrease the number of free parameters, some relations among the fitting Gaussians have been established a priori (Popović \& Mediavilla 1997). Namely, the three Gaussians representing two narrow OIII lines and a narrow $\mathrm{H}_{\beta}$ component are preconditioned to have the same systemic redshift $(z=0.03248$, according to Foltz et al. 1983), and the intensity ratio of the two OIII lines has been supposed to be 1:3.03. Also, the ratio of the full widths of these two lines and narrow $\mathrm{H}_{\beta}$ component are fixed in proportion with their wavelengths as

$\frac{W_{\mathrm{H}_{\beta}}}{4861}=\frac{W_{4959}}{4959}=\frac{W_{5007}}{5007}$

where $W_{\mathrm{H}_{\beta}}$ is width of $\mathrm{H}_{\beta}$ narrow component, $W_{4959}$ and $W_{5007}$ are widths of the two $\operatorname{OIII}(4959,5007)$ lines.

For the $\mathrm{H}_{\beta}$ line region $\left(\mathrm{H}_{\beta}+\mathrm{OIII}\right.$ lines $)$, we obtained the best fit with six Gaussians (Fig. 1) and one "shelf" component. During the considered period, in all groups, $\mathrm{H}_{\beta}$ can be resolved into four components. Three of them are broad and one narrow. The broadest Gaussian covers two other broad Gaussians which are displaced from the center of the $\mathrm{H}_{\beta}$ line toward the blue and red side.

Besides the three broad Gaussians closely connected with $\mathrm{H}_{\beta}$ a wide multi-peaked structure (the shelf) can be noticed at the bottom. The origin of the red shelf in Akn 120 and other AGN's $\mathrm{H}_{\beta}$ line has been discussed in several papers (Foltz et al. 1983; Mayers \& Peterson 1985; Stirpe et al. 1989; van Groningen \& de Bruyn 1989; Korista 1992; Doroshenko et al. 1999). Kollatschny et al. (1981) investigated the combined UV and optical spectra of Akn 120, and noticed that UV and optical Fe II emission in Akn 120 is very strong. Moreover, they found that Akn 120 shows unusually strong Fe II emission in the UV spectra. On the other hand, calculations performed by Joly (1988) indicate that multiplets 25 and 36 may have large contributions in the red wing of $\mathrm{H}_{\beta}$. It was our reason to accept the red shelf Fe II template which has been proposed by Korista (1992). We have supposed that the red shelf is composed of nine Fe II lines belonging to the multiplets 25, 36 and 42 . We took the relative strength of these lines from Korista (1992) and we supposed that all Fe II lines originate from the same conditions (in the same region), meaning that all of them should have the same $W / \lambda$ and shift.

All other lines are also decomposed into a suitable number of Gaussians.

\section{The shape of broad lines}

\subsection{Hydrogen lines}

In order to see the structure of the central part of Akn 120 and to approach a model which can fit the shapes of $\mathrm{H}_{\beta}$ line from the ten periods, we have first analysed the averaged profile of $\mathrm{H}_{\beta}$ as well as averaged profiles of $\mathrm{Ly}_{\alpha}$ and $H_{\alpha}$ line. In Figs. 1 and 2 one can see that either the $\mathrm{H}_{\beta}$ or $\mathrm{H}_{\alpha}$ broad line can be decomposed into three broad 
Table 1. Time-distribution and number of the observed spectra

(in Heliocentric Julian Days - HJD.)

\begin{tabular}{|c|c|c|c|c|c|c|c|c|c|c|}
\hline group & 1 & 2 & 3 & 4 & 5 & 6 & 7 & 8 & 9 & 10 \\
\hline $\begin{array}{c}\text { from HJD } \\
2440000+\end{array}$ & 3435 & 3790 & 4173 & 4522 & 4881 & 5263 & 5619 & 5997 & 6853 & 7832 \\
\hline $\begin{array}{c}\text { till HJD } \\
2440000+\end{array}$ & 3574 & 3934 & 4289 & 4672 & 5054 & 5412 & 5734 & 6088 & 7124 & 7944 \\
\hline $\begin{array}{c}\text { mean HJD } \\
2440000+\end{array}$ & 3494 & 3879 & 4229 & 4577 & 4958 & 5331 & 5695 & 6058 & 7014 & 7888 \\
\hline $\begin{array}{c}\text { numbers of } \\
\text { spectra }\end{array}$ & 14 & 14 & 9 & 5 & 15 & 12 & 11 & 6 & 6 & 4 \\
\hline
\end{tabular}

Gaussian components. It is interesting that in both lines we have similar decomposition, i.e. the broadest component covers the other two broad components which are split in the same way in both lines. In addition, the blue components are more intensive that the red ones.

In the case of the averaged $\mathrm{H}_{\beta}$ line (Fig. 1), the redshift of the blue component is 0.0310 , and width is about $2400 \mathrm{~km} \mathrm{~s}^{-1}$, the red component is shifted to 0.0405 and has a width of about $1200 \mathrm{~km} \mathrm{~s}^{-1}$. The redshift of the broadest component is 0.0382 , and its width is about $5600 \mathrm{~km} \mathrm{~s}^{-1}$.

Also, $\mathrm{H}_{\alpha}$ can be decomposed into four components (Fig. 2) where three components are broad and one narrow. Near the observed wavelength $6800 \AA$, one of the two narrow forbidden N II lines $(\lambda \lambda 6548 \AA$ and $6584 \AA)$ can be found. The other one, three times weaker, is blended with $\mathrm{H}_{\alpha}$ near position of the narrow $\mathrm{H}_{\alpha}$ component. The broadest line is shifted to $d=0.0345$, and has the width of about $4900 \mathrm{~km} \mathrm{~s}^{-1}$. The blue component is shifted by 0.0292 , and its width is about $2100 \mathrm{~km} \mathrm{~s}^{-1}$; the red component is redshifted by 0.0374 , and has a width of about $1600 \mathrm{~km} \mathrm{~s}^{-1}$.

It can be seen from Figs. 1 and 2 that the $\mathrm{H}_{\alpha}$ has one narrow peak with a width of about $590 \mathrm{kms}^{-1}$, and $\mathrm{H}_{\beta}$ also has one narrow component with a width of about $450 \mathrm{~km} \mathrm{~s}^{-1}$.

The $\mathrm{Ly}_{\alpha}$ line allows us to probe the conditions in the very deep regions of the AGN, where plasma undergoes very dynamic changes and turbulence. Consequently, the red component is the broadest (width about $w \approx$ $6600 \mathrm{~km} \mathrm{~s}^{-1}$, shift is $d \approx 0.041$ ), but with the smallest intensity. Even the blue peak is broader $\left(w \approx 2900 \mathrm{~km} \mathrm{~s}^{-1}\right.$ and $d \approx 0.031)$ than the central one $\left(w \approx 1000 \mathrm{~km} \mathrm{~s}^{-1} d \approx\right.$ $0.0328)$, see Fig. 3. The central line, unlike $\mathrm{H}_{\alpha}$ and $\mathrm{H}_{\beta}$, is the most intensive component and, having the smallest line width, originates in the region with the smallest velocity dispersion. It is evident that some absorption is present in the red wing of the line, but as we are dealing only with origin and behavior of the broad lines, in this paper we do not describe it in detail.

\subsection{CIII, CIV, and MgIl line}

The CIII(1909) line (Fig. 4) also shows three broad components $\left(w_{1} \approx 3600 \mathrm{~km} \mathrm{~s}^{-1}, d \approx 0.03535 ; w_{2} \approx 1600 \mathrm{~km} \mathrm{~s}^{-1}\right.$ $d \approx 0.0317 ; w_{3} \approx 1550 \mathrm{~km} \mathrm{~s}^{-1}, d \approx 0.0212$ ), among which the central is the most intens and the red is the widest. In this line the blue component is of about equal relative intensity as the red one - unlike the situation in the previously discussed lines.

The CIV line (Fig. 5) also can be decomposed into three Gaussian broad components $\left(w_{1} \approx 4800 \mathrm{~km} \mathrm{~s}^{-1}, d \approx\right.$ $0.0336 ; w_{2} \approx 2300 \mathrm{~km} \mathrm{~s}^{-1} d \approx 0.0324 ; w_{3} \approx 1100 \mathrm{~km} \mathrm{~s}^{-1}$, $d \approx 0.0321$ ), but here there is no significant difference between shifts of the red, central and blue Gaussian component, except the fourth (smallest) Gaussian, which is within the level of noises which we neglect.

$\operatorname{MgII}(2798)$ line (Fig. 6) is blended with many $\mathrm{Fe}$ lines, but it still could be resolved in the same way as the other lines, showing three broad components $\left(w_{1} \approx\right.$ $2300 \mathrm{kms}^{-1}, d_{1} \approx 0.03346 ; w_{2} \approx 5800 \mathrm{kms}^{-1}, d_{2} \approx$ $\left.0.05148 ; w_{3} \approx 3960 \mathrm{~km} \mathrm{~s}^{-1}, d_{3} \approx 0.09203\right)$. Also, one very broad component $\left(w \approx 8500 \mathrm{~km} \mathrm{~s}^{-1}, d \approx 0.020516\right)$ is present which probably comes from Fe I and Fe II blends in the UV.

All these lines are different from the Balmer ones in three aspects: 1 ) there are no narrow components; 2) the central components dominate by their relative intensity and not by the integrated radiation energy involved; and 3 ) the ratio of blue/red components is not always in favor of the blue; in the C III line, the components are very much equal, in the $\mathrm{C}$ IV line the blue component is much weaker than the red (although the distinction of their identities here is not certain).

As far as the blue/red component ratio is concerned, one can notice a sequence of increasing dissimilarity of the profile shape of these lines with respect to the Balmer ones: H I, Mg II, C III and C IV. Their respective ionization potentials follow the same sequence: $13.6 \mathrm{eV}, 15.0 \mathrm{eV}$, $47.9 \mathrm{eV}$ and $64.5 \mathrm{eV}$, respectively. This fact can indicate a stratification of the corresponding emissivities within the ionizing radiation field.

The similar Gaussian-decomposition structure in all observed spectral lines (expect, perhaps, the CIV line where the grouping of the three relevant Gaussians around 


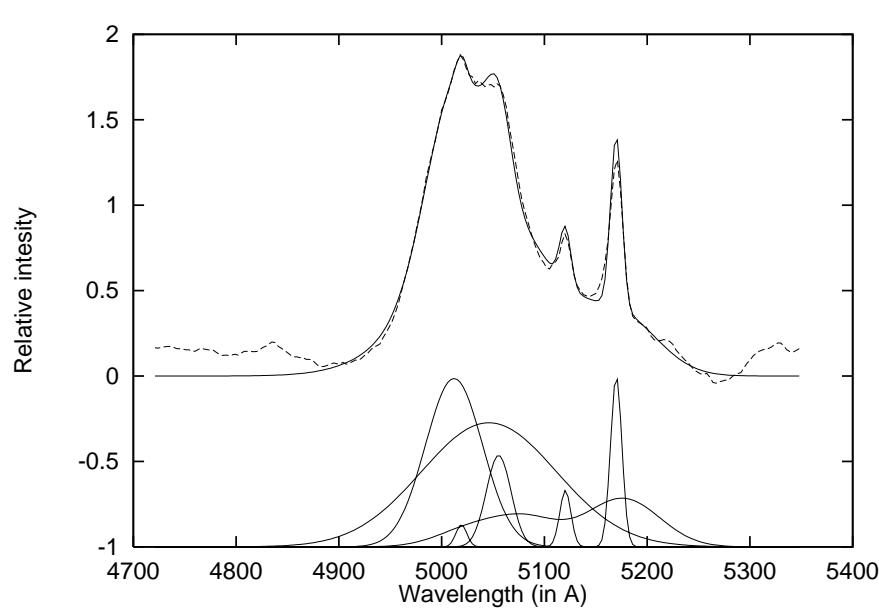

Fig. 1. Decomposition of the averaged shape of the $\mathrm{H}_{\beta}$ line. The dashed line represents observations and solid line the line obtained by Gaussian decomposition. The Gaussian components are presented below

a similar wavelength is unusual and unexpected) may be explained by the origin of the lines from the same BLR or the same BLR components (e.g. BLR1 and BLR2, see Gaskell 1999) but involving different radial distances from the central, ionizing, source.

\subsection{The $H_{\beta}$ variation}

In Fig. 7, we present all ten observed $\mathrm{H}_{\beta}$ profiles and an averaged profile obtained from these considered profiles (full line in Fig. 7).

The shape of $\mathrm{H}_{\beta}$ shows very strong variation (Fig. 7), especially in the central part of the line. Here one should notice three parts; sometimes a shoulder in the blue part of profile, a blue and a red peak. Low-quality data were rejected. The other problem is the very weak OIII (5007) line which we used for flux calibration of $\mathrm{H}_{\beta}$ line region.

As one can see from Fig. 7 , the $\mathrm{H}_{\beta}$ line has two peaks displaced toward the red and blue with respect to the redshifted center of the line. The highest scatter of the profiles is within the $\mathrm{H}_{\beta}$ core. This we take as real change in the $\mathrm{H}_{\beta}$ flux and profile shape over time. The moderate scatter of the curves near both edges of the interval of wavelengths may partly reflect changes in two Fe II emission complexes around the observed wavelengths $4727 \AA$ and $5430 \AA$. However, it also can be caused by some uncertainties in the reduction of the continuum.

In all observed groups (ten groups in Table 1 ) the mean spectra show the complex shape of the $\mathrm{H}_{\beta}$ line of Akn 120 . The profiles can be decomposed into four components, where three of them are broad. Also, one red shelf composed of nine Fe II lines from multiplets 25, 36 and 42 is present in the red wing of $\mathrm{H}_{\beta}$.

The temporal changes of the shifts, widths and sizes $\left(I_{0} w / \lambda\right)$ of the three broad Gaussians of the $\mathrm{H}_{\beta}$ line are shown in Figs. 8-10.

As shown in Fig. 8, the shift of the blue and red Gaussian components show similar behaviour during time.

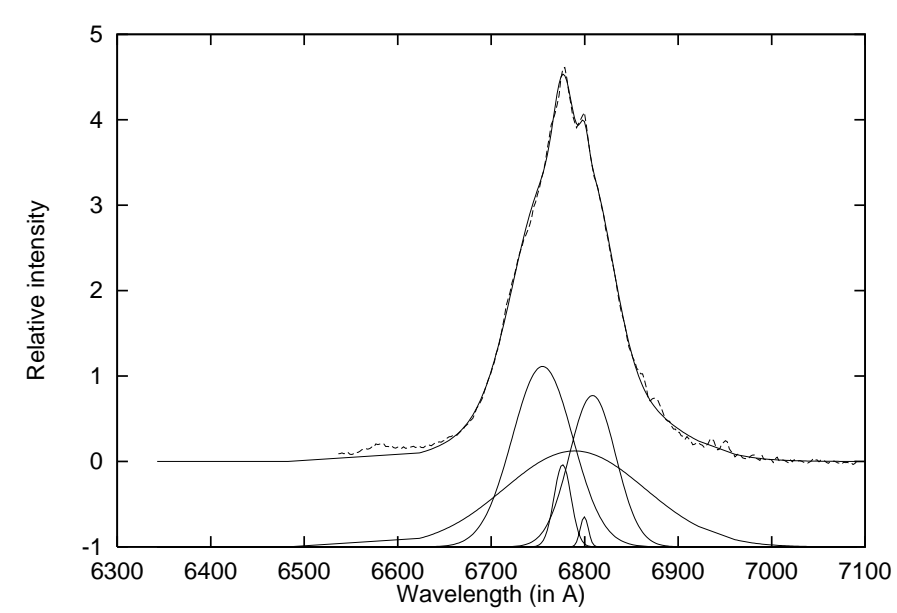

Fig. 2. Decomposition of the averaged shape (dashed line) of the $\mathrm{H}_{\alpha}$ line and the best fit (solid line). The Gaussian components are presented below

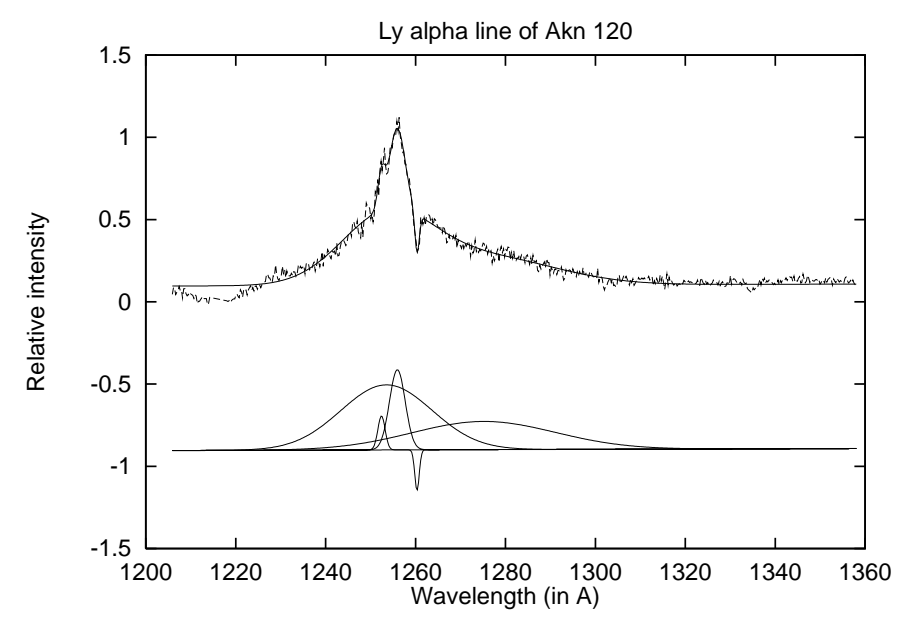

Fig. 3. Decomposition of the averaged shape of the the $\mathrm{Ly}_{\alpha}$ line. The notation is the same as in Figs. 1 and 2

Also, the shift of these two components is relatively stable, while the shift of the broadest (central) component shows significant variation. On the other hand, the widths (Fig. 9), which indicate the random velocities in the regions, show very clearly the existence of three regions or separated velocity fields. Two of them (shifted red and blue) are quite stable, while the broadest shows very large changes. From the changes in intensity of the components (Fig. 10) one can see that the broadest and blue components display a stronger variability in intensity than does the red one. It may indicate that these regions are closer to the center of AGN. The structure of the emitting region in Akn 120 may be such that we observe two regions which are in the streams (as proposed by Zheng et al. 1990), and one central region which is close to or belongs to an emitting disk (as proposed by Allion et al. 1988). It seems that the core of the $\mathrm{H}_{\beta}$ line originates in the two shifted regions and the wings arise from the disk and a central, highly turbulent region which may be disk-like. 


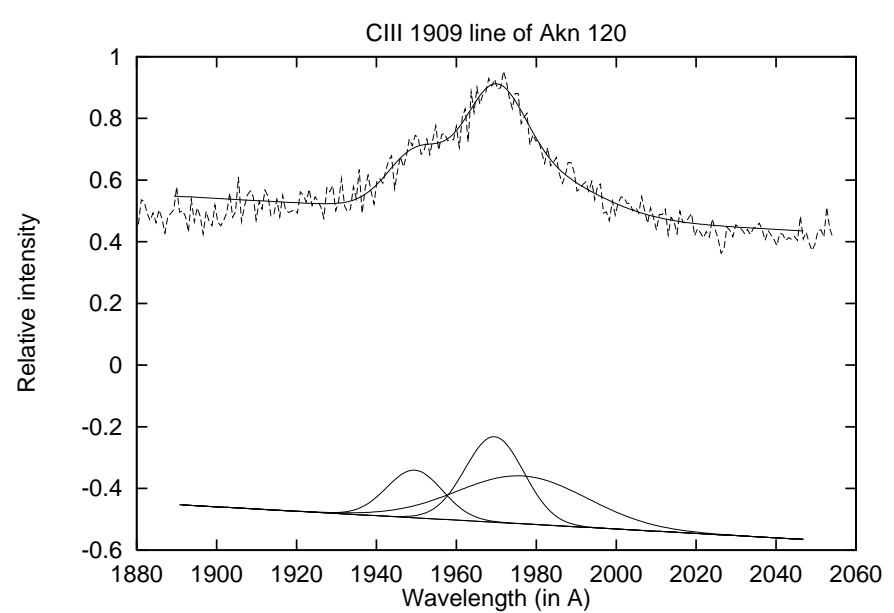

Fig. 4. Decomposition of the averaged shape of the CIII[1909] line. The notation is the same as in Fig. 1

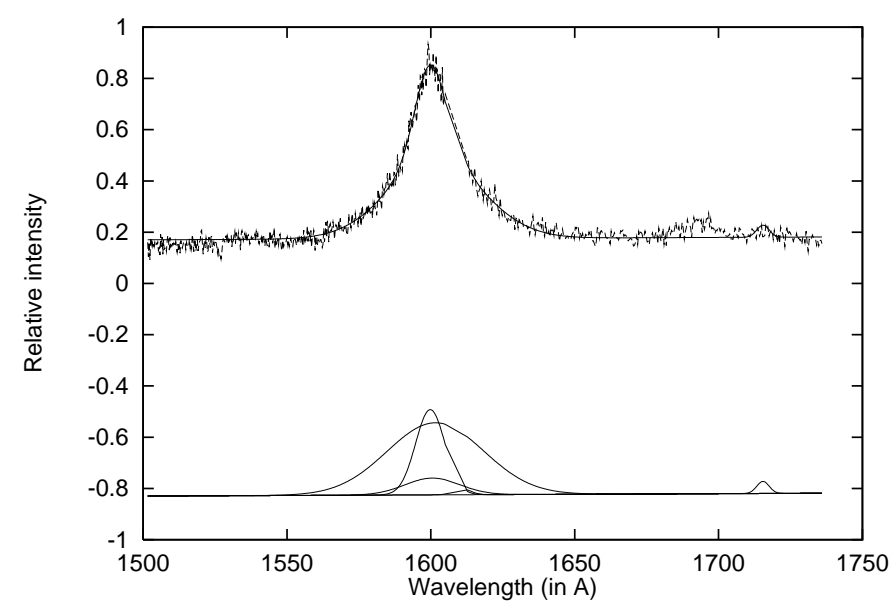

Fig. 5. Decomposition of the averaged shape of the CIV[1549] line. The notation is same as in Fig. 1

\section{The model of Akn 120 BLR}

In all the considered periods, the $\mathrm{H}_{\beta}$ line has two (sometimes three) peaks. The nature of the double-peaked lines in AGNs has been discussed in several papers (see e.g. Chen \& Halpern 1989; Chen et al. 1989; Zheng et al. 1990; Zheng et al. 1991; Goad \& Wanders 1996; Popović et al. 1998b; Gaskel et al. 1983, 1996). The origin of double peaked lines in AGNs can be explained by:

a) emission by the accretion disk or emission from spiral shock waves within a disc (Chen et al. 1989; Chen \& Halpern 1989);

b) emission from the oppositely-directed sides of a bipolar outflow (Zheng et al. 1990; Zheng et al. 1991);

c) emission from a spherical system of clouds in randomly inclined Keplerian orbits, illuminated anisotropically from the center (Goad \& Wanders 1996); and

d) emission from a binary black hole system (Gaskell 1983, 1996; Popović et al. 1998b).

Each of the proposed mechanisms can explain the double peaked lines, but the accretion disk model is most often used to explain double-peaked line profiles, because

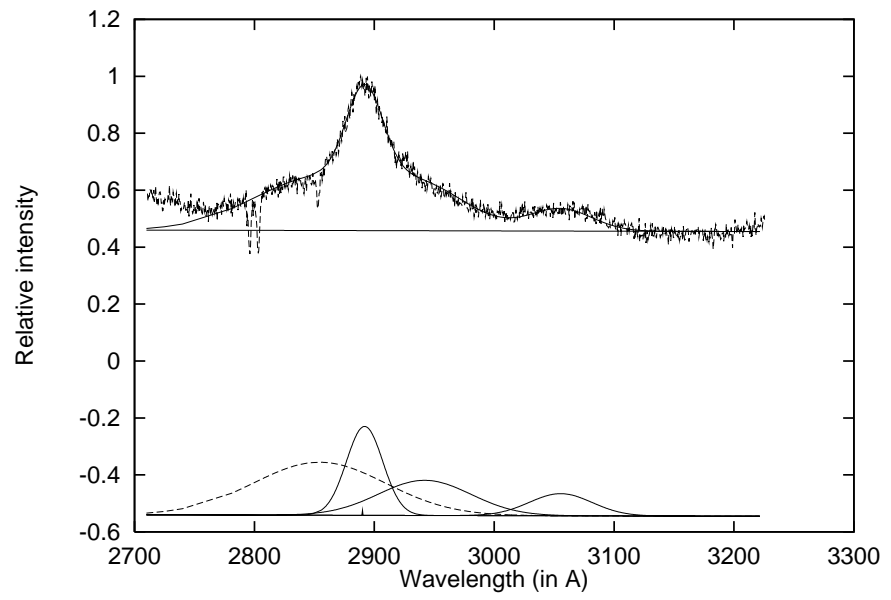

Fig. 6. Decomposition of the averaged shape of MgII[2798] line. The notation is same as in Fig. 1. The dashed Gaussian component below may be contributed partly by Fe blends

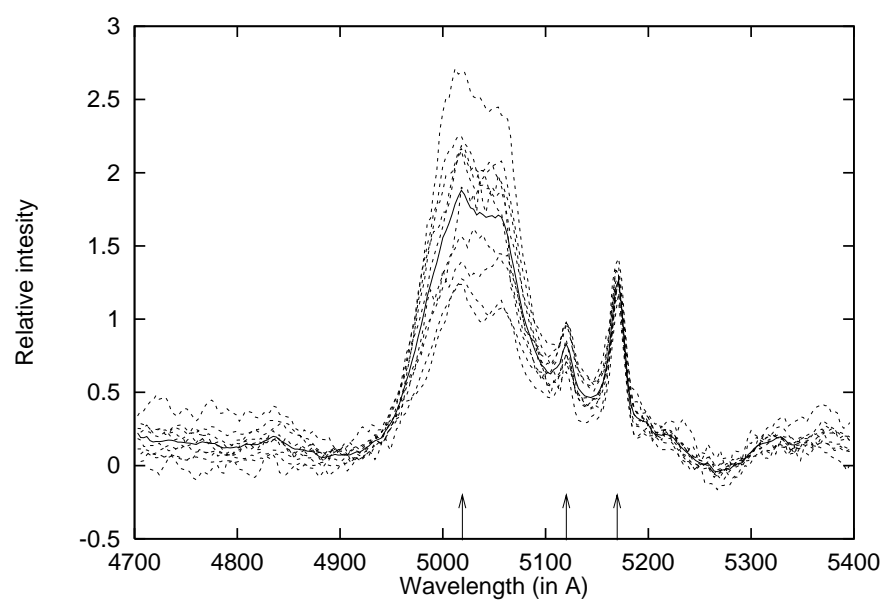

Fig. 7. The shape of $\mathrm{H}_{\beta}$ in all considered periods (broken lines) and an averaged profile for $\mathrm{H}_{\beta}$ from these periods (full line). The arrows show the positions of cosmologically redshifted $\mathrm{H}_{\beta}$ and OIII (4959 and 5007) lines

this model fits well with the widely accepted picture of an AGN, where the "central engine" consists of a massive black hole fueled by an accretion disk. Moreover, the observations over a wide spectral range (X, UV, optical) indicate that an accretion disk should be present in the central part of most AGNs. The geometry of the Akn 120 emitting region has been discussed several times (Allion et al. 1988; Zheng et al. 1990; Doroshenko et al. 1999, etc.) and two models have been considered: 1) the disk model by Allion et al. (1988) and 2) the double-stream model by Zheng et al. (1990). Both of these models can explain the double peaked lines, but in choosing the model one has to take into consideration line profiles as well as their variations.

We started from the following: 1) the long-period variation of $\mathrm{H}_{\beta}$ indicated that three emission regions in the central part of Akn 120 exist in all periods considered, Figs. 8-10. The variation in the blue and central components, may indicate that they come from regions which 


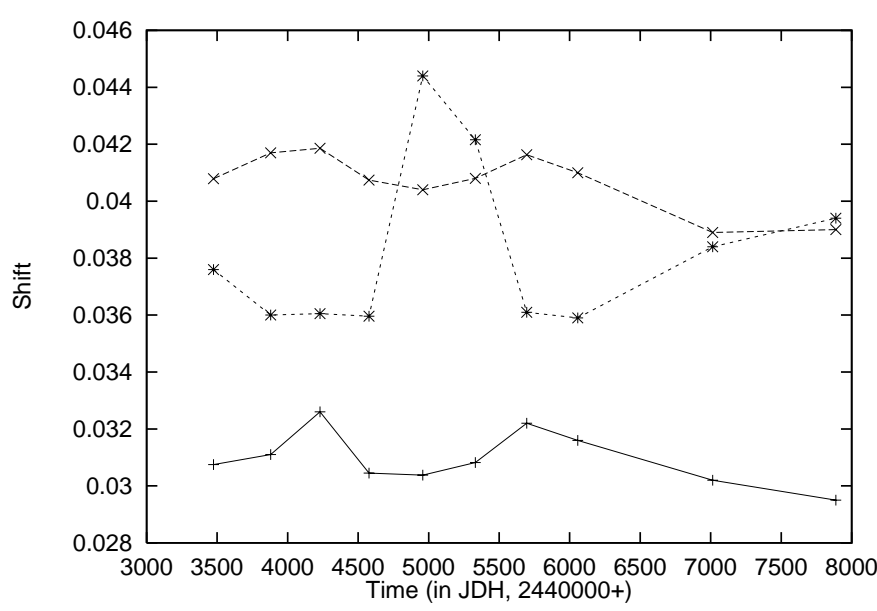

Fig. 8. Redshifts of the three broad Gaussians for 10 groups of Akn 120 spectra $\left(+=\right.$ blue peak, $x=$ red peak, ${ }^{*}=$ the broadest Gaussian)

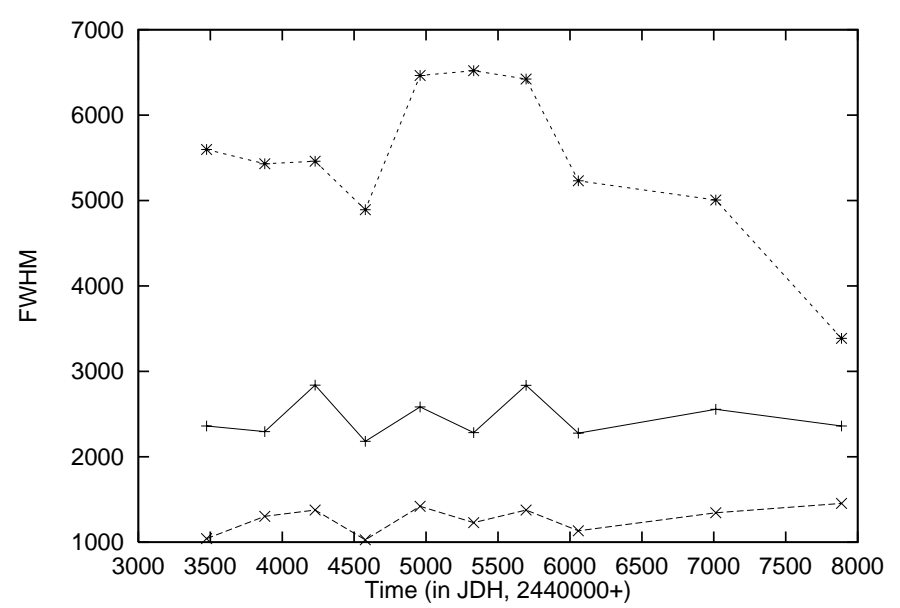

Fig. 9. Same as in Fig. 11, but for the Gaussians' width (in $\mathrm{km} \mathrm{s}^{-1}$ )

are closer to the central mass, whereas the red component may be produced by a stream which is partly obscured by the disk and the central region, and we see only part of the "red stream"; 2) the shape of Ly $\mathrm{L}_{\alpha}$ indicates (especially its wings) that the radiation of the disk is present in this line. Taking these two facts into account, we conclude that a model with an accretion disk and two regions symmetrical to the disk may well fit the line profiles of the hydrogen lines.

Also, the blue-boosted and redshifted broad Gaussian components in all Akn 120 lines considered (except the C IV line) may indicate that an accretion disk is present. But, concerning the variation on longer time-scales, the bumps in the sides of lines vary independently (Gaskell \& Snedden 1999). The disk model cannot explain the shape of all the spectral lines (see e.g. Fig. 7). Here, we apply the disk model (Chen \& Halpern 1989) to explain the wing shapes. First, we subtracted the narrow lines from $\mathrm{H}_{\alpha}$, an absorption line from $\mathrm{Ly}_{\alpha}$ and narrow $\mathrm{O}$ III lines as well as an Fe II template from $\mathrm{H}_{\beta}$. Then, we fitted these line profiles by a disk model (Chen \& Halpern 1989; Chen

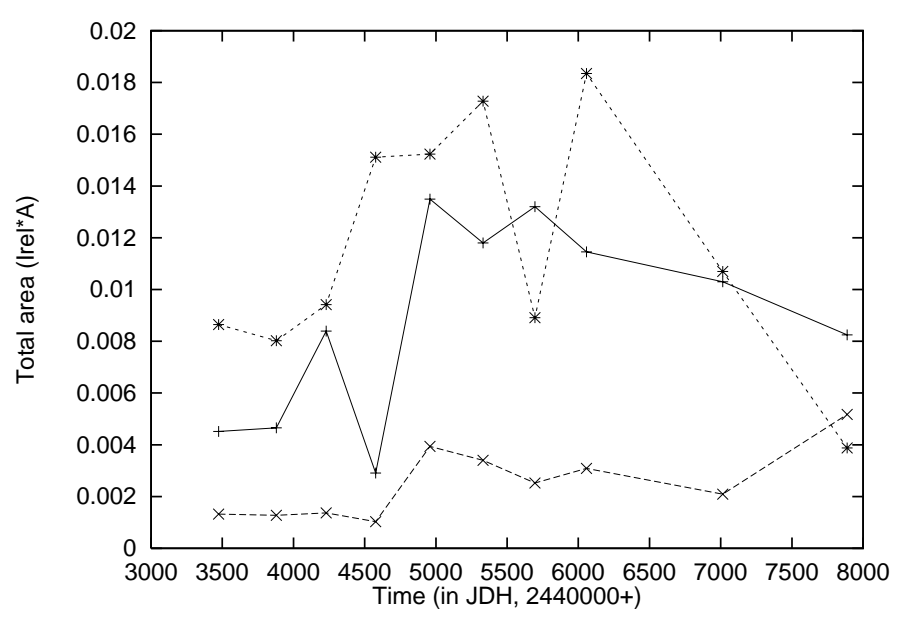

Fig. 10. The areas under the broad Gaussian components of the $\mathrm{H}_{\beta}$ line as a function of time $(+=$ blue peak, $x=$ red peak, $*=$ the broadest Gaussian)

et al. 1989) plus one or two Gaussians. The emissivity of the disk as a function of radius is given as

$\epsilon=\epsilon_{0} R^{-p}$,

where the power index $p=3$. Also, we express the disk dimension in gravitational radii $\left(R_{\mathrm{g}}=G M / c^{2}\right.$, where $G$ is the gravitational constant, $M$ is the mass of the central black hole, and $c$ is velocity of light).

The results of the fit are presented in Figs. 11-13. We fitted the $\mathrm{Ly}_{\alpha}$ profile with the disk model with parameters: $i \approx 10^{\circ}, R_{\text {inn }} \approx 120 R_{\mathrm{g}}$ and $R_{\text {out }} \approx 1150 R_{\mathrm{g}}$ and one Gaussian component, which indicates a random velocity, $w$, of $1800 \mathrm{~km} \mathrm{~s}^{-1}$. For Balmer lines, we could not fit the lines with the disk model and one Gaussian component. We propose that the the central part of these lines corresponds to the other two regions. Balmer lines were fitted with the next parameters of the disk: $i=10^{\circ}$ for both lines and for $\mathrm{H}_{\alpha}-R_{\mathrm{inn}} \approx 200 R_{\mathrm{g}}$ and $R_{\text {out }} \approx 1000 R_{\mathrm{g}}$, the blue Gaussian component has $w \approx 1900 \mathrm{~km} \mathrm{~s}^{-1}$ and the red has $w \approx 1500 \mathrm{~km} \mathrm{~s}^{-1}$; for $\mathrm{H}_{\beta}-R_{\text {inn }} \approx 165 R_{\mathrm{g}}$ and $R_{\text {out }} \approx 1000 R_{\mathrm{g}}$, the blue Gaussian component has $w \approx$ $2100 \mathrm{~km} \mathrm{~s}^{-1}$ and the red has $w \approx 1700 \mathrm{~km} \mathrm{~s}^{-1}$.

\section{Conclusion}

In general, we accept the idea that there are two distinct components of a BLR (Gaskell 1999) or that some other type of stratification exists in a BLR (Korista 1992)

As long as we remain in the Gaussian-decomposition approach, we are ready to interpret the two broad Gaussians corresponding to the blue and red Balmer line profile peaks as originating from somewhere outside the accretion disk - probably from a biconical BLR configuration (BLR1 and BLR2 in Fig. 14). In $\mathrm{H}_{\beta}$ the blue Gaussian is always stronger and the two change independently. The third, the broadest and the most stable Gaussian, may represent the energy irradiated by the accretion disk. However, with its predestined mono-peaked 


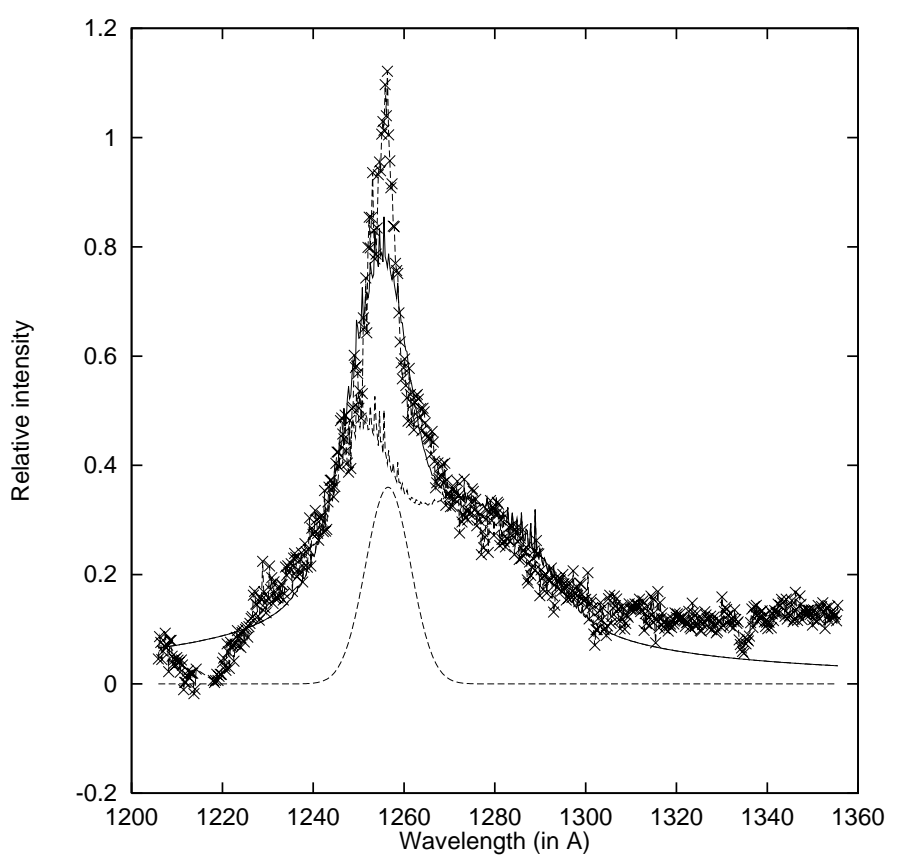

Fig. 11. Observed $\mathrm{Ly}_{\alpha}$ of Akn $120(x-x-)$, fitted by the disk model (dashed line) plus one Gaussian (dashed line)

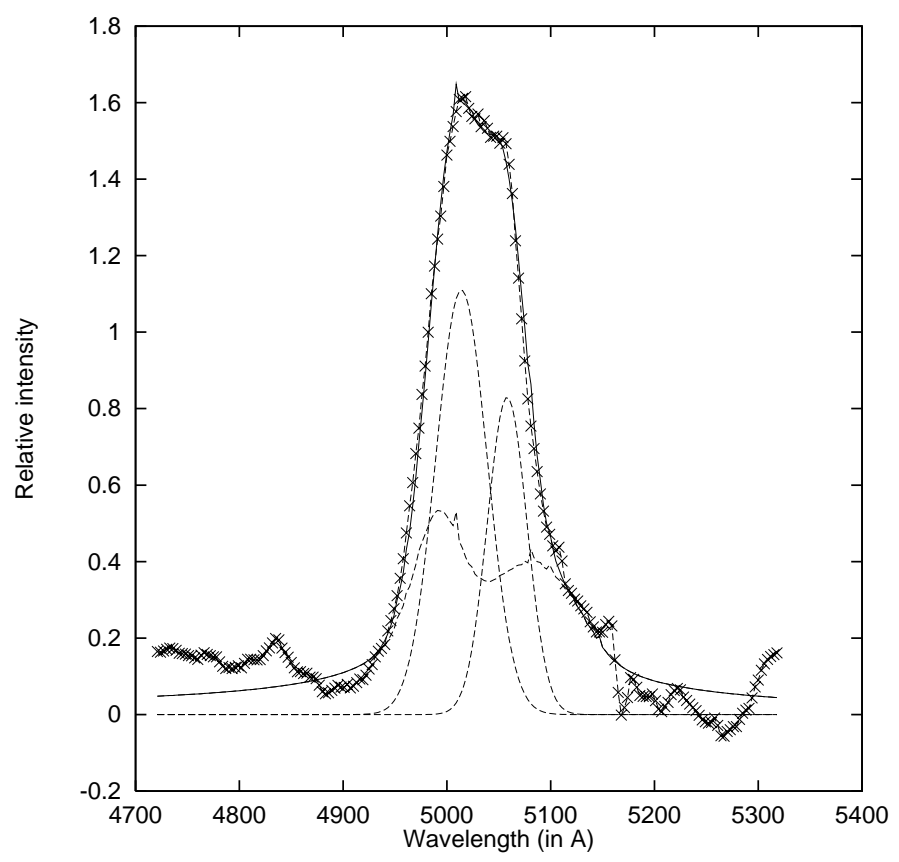

Fig. 12. Same as in Fig. 10, but for $\mathrm{H}_{\beta}$

shape a Gaussian cannot reflect the detailed disk profile: "blue boosted, red shifted" (e.g. Stuchlik 1998). Only in the unique case of an extremely turbulent accretion disk do the two profile peaks merge (van Groningen 1983) and a Gaussian may, in an approximation, represent the disk component of a spectral line profile.

The main conclusion which can be drawn from the present analysis of the $\mathrm{H}_{\beta}, \mathrm{H}_{\alpha}$, Ly ${ }_{\alpha}$, CIII[1909], CIV[1549] and $\mathrm{MgII}[2798]$ lines, is that these lines mainly exhibit

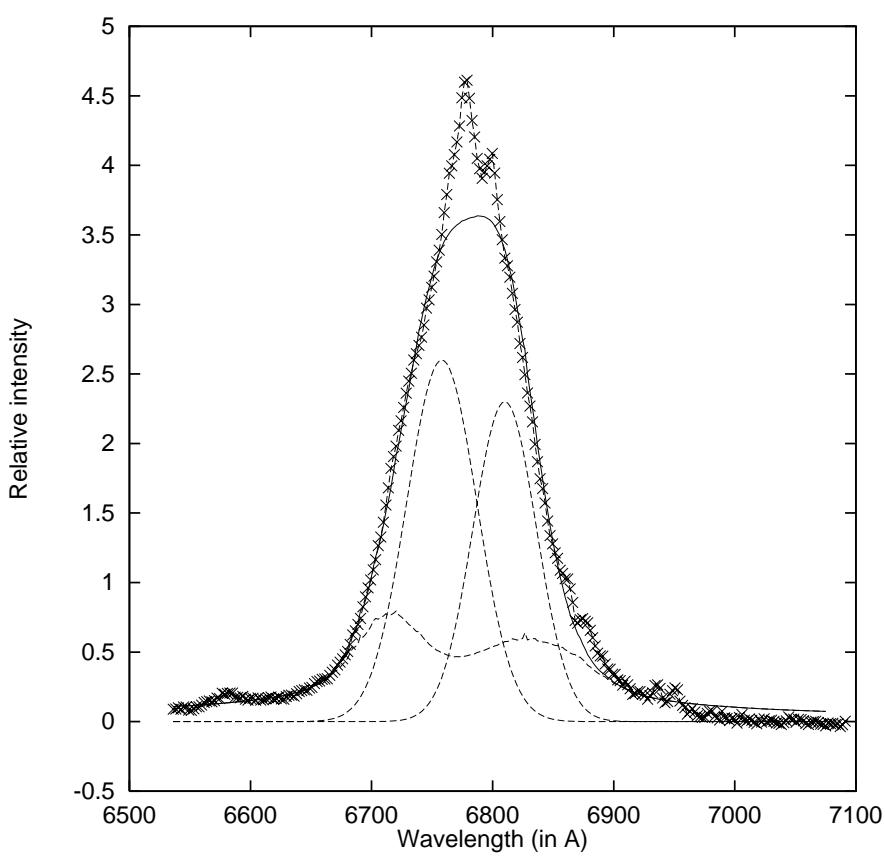

Fig. 13. Same as in Fig. 10, but for $\mathrm{H}_{\alpha}$

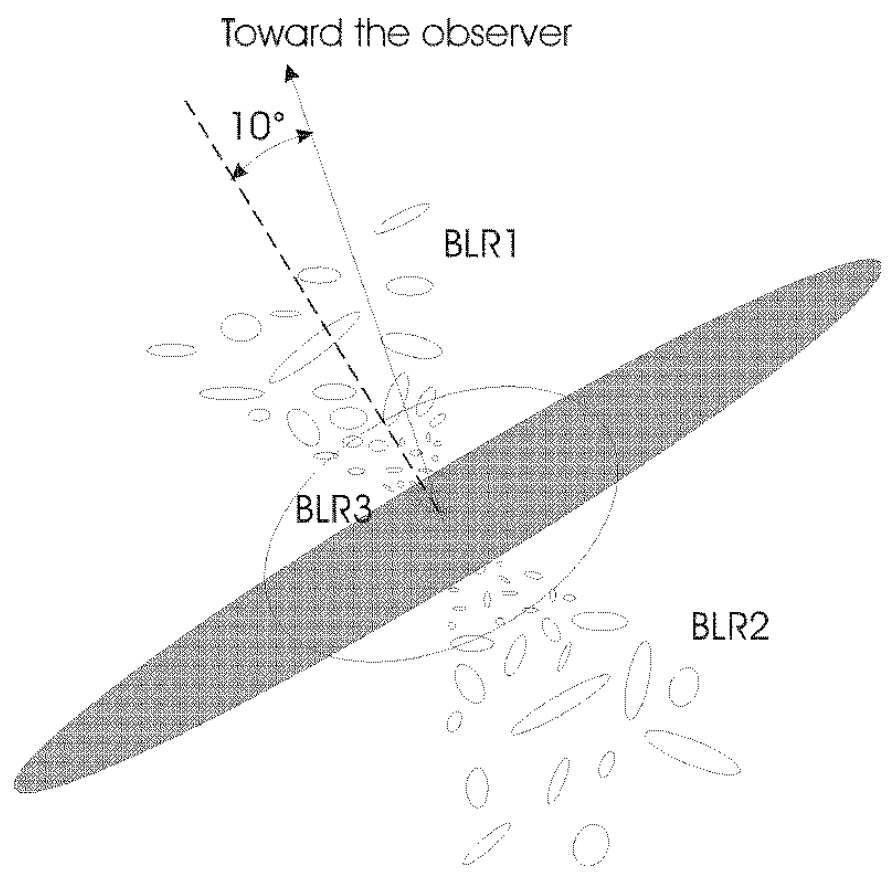

Fig. 14. The scheme of the proposed model for Akn 120

blue boosted - red shifted behavior. It has led us to propose the disk model (see van Gronongen 1983; Allion 1988; Chen \& Halpern 1989) which can describe the line wings and the double-stream model. The $\mathrm{Ly}_{\alpha}$ can be produced in the inner parts of this disk while its core originates in the stream near the central mass and the other component cannot be seen. The $\mathrm{H}_{\beta}$ and $\mathrm{H}_{\alpha}$ originate from the outer parts of the same assumed disk as well as in outer part of the streams. The wings of $\mathrm{H}_{\alpha}, \mathrm{H}_{\beta}$ and $\mathrm{Ly}_{\alpha}$ can be fitted by a disk model. The parameters of the disk are: $i=10^{\circ}, R_{\text {inn }} \approx 120 R_{\mathrm{g}}$ and $R_{\text {out }} \approx 1200 R_{\mathrm{g}}$. The scheme 
of the emitting region in Akn 120 used in our analyse is given in Fig. 14. As one can see from the Fig. 14, we propose a model with a disk, or a related (BLR3) central region (probably disk-like) which surrounds a part of the disk, and also two regions located in the axial streams (BLR1 and BLR2), where one of them (redshifted, BLR2) is partly obscured by the accretion disk and central region. Taking into account the estimated mass for Akn 120 ( $\left.M=1.9310^{8} M_{\odot}\right)$ given by Wandel et al. (1999) we can obtain the dimension of the radiating disk of about 12 light days, which is in agreement with the estimation given in Wandel et al. (1999), where the estimated dimension of Akn 120 BLR is about 11.748 light days.

Acknowledgements. This work was supported by the Ministry of Science and Technology of Serbia through the project "Astrometrical, Astrodynamical and Astrophysical Researches" and The Federal Ministry of Developement, Sciences and Environmental Protection of Yugoslavia. L. Č. P. and N. S. are grateful to Dr. Nina Polosuhkina and Valya Doroshenko for help and suggestions. L. Č. P. acknowledges support by the Berlin Brandenburg Academy of Science and Humanities in the framework of its Balkan-Initiative and thanks the AIP for the hospitability during his stay at the Institute.

\section{References}

Allion, D., Boisson, C., \& Pelat, D. 1988, A\&A, 200, 17

Chen, K., Halpern, J. P., \& Filippenko, A. V. 1989, ApJ, 339, 115

Chen, K., \& Halpern, J. P. 1989, ApJ, 344, 115

Doroshenko, V. T., Sergeev, S. G., Pronik, V. I., \& Chuvaev, K. K. 1999, Astron. Lett., 25, 9, 569

Foltz, C. B., Wilkes, B. J., \& Peterson, B. M. 1983, AJ, 88, 1702

Gaskell, C. M. 1983, Proc. 24th Liège Intern. Ap. Colloquium, Univ. de Liège, Liège, 473

Gaskell, C. M. 1996, ApJ Lett., 464, 107

Gaskell, C. M. 1999, in ASP Conf. Ser. 175, Structure and Kinematics of Quasar Broad Line Regions ed. G. M. Gaskell, V. N. Brandt, M. Dietrich, D. Dultain-Hacyan, \& M. Eracleous, 423
Gaskell, M. C., \& Snedden, A. 1999, in ASP Conf. Ser. 175, Structure and Kinematics of Quasar Broad Line Regions ed. C. M. Gaskell, W. N. Brandt, M. Eracleous, M. Dietrich, \& D. Dultzin-Hacyan, 157

Goad, M. \& Wanders, I. 1996, ApJ, 469, 113

Jackson, N. \& Browne, I. W. A. 1989, MNRAS, 236, 97

Joly, M. 1988, A\&A, 192, 87

Kollatschny, W., Schleicher, H., Fricke, K. J., \& Yorke, H. W. 1981, A\&A, 104, 198

Korista, K. T. 1992, ApJSS, 79, 285

Meyers, K. A., \& Peterson, B. M. 1985, PASP, 97, 734

Osterbrock, D. E. 1990, Astrophysics of Gaseous Nebulae and Active Galactic Nuclei, Mill Valley, California

Peterson, B. M. 1993, PASP, 105, 247

Peterson, B. M., \& Cota, S. A. 1987, AJ, 94, 7

Peterson, B. M., \& Gaskell, C. M. 1991, ApJ, 368, 152

Peterson, B. M., Meyers, K. A., Capriotti, E. R, et al. 1985, ApJ, 292, 164

Peterson, B. M., Wanders, I., Bertram, R., et al. 1998, ApJ, 501,82

Peterson, B. M., Korista, K. T., \& Wagner, R. M. 1989, AJ, 98,500

Marziani, P., Calvani, M., \& Sulentic, J. W. 1992, ApJ, 393, 658

Popović, L. Č., \& Mediavilla, E. 1997, Publ. Astron. Obs. Belgrade, 57, 95

Popović, L. Č., Trajković, N., Kubičela, A., et al. 1998a, Publ. Astron. Obs. Belgrade, 61, 139

Popović, L. Č., Pavlović, R., Živkov, V., Djurašević, G., \& Kubičela, A. 1998b, 19th SPIG Contributed papers ed. N. Konjević, M. Ćuk \& I. Videnović, Faculty of physics, University of Belgrade, Belgrade, 639

Stirpe, G. M., van Groningen, E., \& de Bruyn, A. G. 1989, A\&A, 211, 310

Stuchlik, Z. 1998, Proc. 20th Stellar Conference, ed. J. Dushek, \& M. N. Zejda, Copernicus Obs. and Planetarium, Brno, 147

van Groningen, E. 1983, A\&A, 126, 363.

van Groningen, E. \& de Bruyn, A. G. 1989, A\&A, 211, 293

Wandel, A., Peterson, B. M., \& Malkan, M. A. 1999, ApJ, 526, 579

Winge, C., Peterson, B. M., Pastoriza, M. G., \& StorchiBergmann, T. 1996, ApJ, 469, 648

Zhang, W., Binette, L., \& Sulentic, J. W. 1990, ApJ, 365, 115

Zheng, W., Veilleux, S., \& Grandi, S. A. 1991, ApJ, 381, 418 